\title{
Stadt der Entfremdung
}

Rezension zu Rowland Atkinson (2020): Alpha City. How London Was Captured by the Super-Rich. London: Verso.

\section{Adrian Kreutz}

Abb. 1 Titel des Buches (Verso Books)

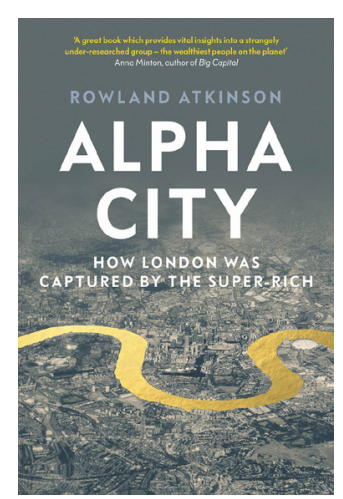

Bei einem Spaziergang durch London, durch die wohlhabendsten als auch die ärmeren Stadtviertel, sieht man die vielen leerstehenden Wohnungen - 125.000, um genau zu sein (Action on Empty Homes 2020) -, die von Investor_innengekauft und zu einem Symbol für diekaputte Wohnungspolitik einer zunehmend ungerechten, fast schon hobbesianischen Gesellschaft geworden sind. Rowland Atkinsons Alpha City (2020) befasst sich eingehend mit der Frage, wie vermögende Privatpersonen als Inkarnationen des Kapitals - zumeist von ,Offshore - das urbane Gefüge Londons veränderten, indem sie andere verdrängten, die Stadt zu ihrer ,Hauptstadt - ihrer ,AlphaStadt'[1] - machten, und was genau dies für die Verdrängten bedeutet.

\section{Was ist eine Alpha-Stadt?}

Eine Alpha-Stadt bildet den Nährboden für eine einzigartige, kraftvolle Verbindung von wirtschaftlichen, sozialen und politischen Interessen. Eine Alpha-Stadt ist eine Stadt von und für das Kapital. Sie ist eingebettet in die (kapitalistische) Weltwirtschaft und kann als ihr Herzstück betrachtet werden. Es ist eine Stadt, die die Logiken von Geld, Märkten und Finanzen verbindet: ein sicherer Hafen für das Kapital in einer destabilisierten Welt (vgl. Beaverstock et al. 1999). Wenn wir den Begriff auch auf vorkapitalistische Zeiten anwenden möchten, mögen einst Venedig, Jericho, Uruk, Babylon, Peking oder Rom den Titel der Alpha-Stadt getragen haben.

Das Globalization and World Rankings Institute unterscheidet Städte nach den Status Alpha++, Alpha+, und Alpha. Neben London hat nur New York Anspruch auf den Alpha++-Status. Seit der Finanzkrise im Jahr 2008 sei jedoch London zur Spitze dieses Systems - eben zur der Alpha-Stadt - geworden, so Atkinson. Durch London fließt mehr Geld als durch alle anderen Orte der Welt, London hat den teuersten Immobilienmarkt und lockt die reichsten Menschen.

Den Weg zur absoluten Alpha-Stadt ebneten die Politiker_innen Londons, die sowohl niedrige Steuern als auch ungezügelte Unternehmensfreiheiten ermöglichten, Formen der Steuervermeidung ignorierten, Zölle senkten, 
Vorschriften anpassten und staatliche Systeme (insbesondere in Bezug auf Stadt- und Wohnungspolitik) eben für das Kapital und nicht für die Bewohner_innen Londons arbeiten ließen.

Im Wesentlichen, so Atkinson, führte die sich seit der Finanzkrise von 2008 stetig potenzierende Neoliberalisierung des Londoner Wohnungsmarktes zu einer kollektiven Entfremdungserfahrung, die sich durch alle sozialen Schichten zieht und auch vor den Superreichen[2] nicht Halt macht.[3] Die Wohlhabenden schotten sich von der Außenwelt ab, die Mittelschicht und die Einkommensschwachen werden aus der Stadt verdrängt[4]: 28 Prozent aller Londoner_innen, also 2,5 Millionen Menschen, leben laut der Stiftung Trust for London (Stand August 2020) in Armut. 9 Millionen Menschen leben in 3,5 Millionen offiziell gemeldeten ,Wohnungseinheiten', wobei 50 Prozent aller Londoner alleinstehend sind, was darauf hinweist, dass ,Wohnungseinheiten' überbewohnt werden. Auf die einkommensstärksten 10 Prozent kommen 32 Prozent des Gesamteinkommens in der Stadt, den wohlhabendsten 20 Prozent aller Londoner_innen gehören 62 Prozent des Gesamtvermögens, das sich zurzeit in London befindet.

Im Mittelpunkt von Atkinsons Buch steht die Beobachtung, dass die relativ kleine Zahl der Superreichen die Spitze eines großen Eisbergs ist, dessen tieferer, vor dem öffentlichen Auge verborgener Einfluss von einer eigennützigen und erfolgreichen Klasse von sogenannten „Befähiger_innen“ (enablers) getragen wird. Diese Schlüsselakteure, die für das Kapital arbeiten, sind in den Finanz-, Immobilien-, Politik- und Wirtschaftssektoren zu finden. In der Alpha-Stadt konzentrieren sich diese intermediären Befähiger_innen (vgl. Beaverstock 2012; Beaverstock et al. 2012).

\section{(Über-)Leben in der Alpha-Stadt}

Vor mehr als fünfzig Jahren bemerkte der britische Schriftsteller George Mikes (1951), London sei eine Art „integriertes Chaos“ (chaos incorporated). Auch heutetrifft das zu. Aus der Perspektiveder Superreichen und deren Befähiger_innen ist dieses Chaos jedoch nicht mehr als ein Hintergrundgeräusch. Wie Atkinson eindrücklich darlegt, haben die Londoner Superreichen ihre Abschottungstechniken perfektioniert (vgl. Atkinson 2006; Koh et al. 2016). Diese lokale Abschottung führt zu einer moralischen Atmosphäre, die sich wie ein soziales Opiat verhält: Sie führt zu einem Eskapismus in abgelegene Orte, von denen aus das Chaos der Metropole nicht sichtbar ist. Atkinson psychologisiert nicht; dennoch können wir den Entfremdungseffekt dieser Trennungspraktiken leicht erahnen.

Verdrängen die Superreichen die Reichen, verdrängen die Reichen die Mittelschicht, und so verschiebt sich die komplette Demographie einer Stadt. Wenig deutlich wird in Atkinsons Buch, dass auch die Spitzenverdiener_innen in der Immobilienbranche, der Politik oder der Stadtplanung auf den Schultern einer weiteren, enabling class' stehen, oft Einwanderer_innen aus Süd- und Osteuropa sowie den ehemaligen Kolonien.[5] Die Ärmsten Londons werden in den Diensten ihrer reicheren Zahlmeister buchstäblich als Geiseln des Kapitals gehalten. Ihre Arbeitsplätze in den Hierarchien der Befähiger_innen, die um eine bestimmte Kerngruppe der Superreichen herum angesiedelt sind, sind vom Geldfluss der Alpha-Stadt abhängig.[6] 
Die Menschen werden von der ,Logik dieses Geldes' gekauft, sagt Atkinson. Dabei merken die Befähiger_innen oft nicht, so Atkinson, dass das Geld wie eine plutokratische Nekrose wirkt: Die Superreichen untergraben ihre eigene Stadt.

Diese Ideologisierung spielt den Bemühungen mächtiger Unternehmensinteressen und Akteure in die Tasche, deren Werkzeuge die politische Finanzierung, einseitige Stadtplanung, einschlägige Thinktanks, das GoldenPassport-Regime[7] und ein blindes Auge für Geldwäsche sind. Das ,gewöhnliche' Leben in der Stadt wird durch den Mangel an Sozialleistungen und Wohnmöglichkeiten, die durch das politische Management zugunsten der kosmopolitischen Elite entstehen, aktiv untergraben.

\section{Trickle-down-Effekte?}

Die Antwort der britischen Regierung und der Verwaltung Londons auf die sich zuspitzende Wohnungskrise besteht in einer weiteren Öffnung und Deregulierung des Wohnungsmarktes sowie massiven Steuersenkungen für Offshore-Käufer und ,Entwickler'. Die Devise: Je mehr gebaut wird, desto besser. Ein solches Ethos untermauert auch die Veränderungen, die in vielen Gemeinden durch die Sanierung von Sozialsiedlungen und den Abriss tausender Häuser herbeigeführt werden. Gebaut werden kaum neue Sozialwohnungen, sondern Luxus-Appartements.[8]

Arme Haushalte und Haushalte mit mittlerem Einkommen bilden den Treibstoff der Alpha-Stadt-Maschine. Dennoch werden sie, während sie arbeiten und zu überleben versuchen, auch zu ihrem Abfallprodukt. Die Nachfrage nach Immobilienkrediten nimmt zu, was dazu beiträgt, dass Immobilienpreise noch schneller steigen. Das bedeutet auch, dass viele Menschen einen zunehmend großen Teil ihres Einkommens für Miete entbehren oder gar die Stadt verlassen müssen.

Der ,Trickle-down-Effekt', der die Devise des ,mehr Bauens ' legitimieren soll, existiert, aber nicht in der Form, wie er von der britischen Regierung herangezogen wird (vgl. Baumann 2013; Sayer 2015). Viele Geringverdiener_innen zahlen steigende Beträge ihres Einkommens für minderwertige private Mietwohnungen. Hohe Wohnungs- und Transportkosten - für jene, die aus der Stadt vertrieben wurden - sind Aspekte des Lebens in einer Stadt, die sich völlig dem Finanzwesen und einem Immobilen-Markt als „globale Reservewährung“ (Goldfarb 2013) verschrieben hat. Der ,Trickle-downEffekt ${ }^{\star}$ des Reichtums existiert, aber er nehme die Form dieser effektiven Belastung des täglichen Lebens an, so Atkinson.

\section{Was tun?}

Atkinsons Beitrag zur Debatte ist vornehmlich deskriptiv, teilweise etwas voyeuristisch. Er fragt: Wie konnte das Kapital, im weitesten Sinne des Wortes, die Stadt erobern (capture)? Wie hat Geld die gebaute Umwelt verändert? In welchen Symbolen des urbanen Raums zeigt sich die Geldmacht? Welche Position nimmt die Alpha-Stadt als Knotenpunkt der globalen Wirtschaft ein? Auf diese Fragen findet Atkinson im Sein und Schein der Superreichen eine Antwort. Dabei erweitert Atkinson den Begriff der Elite 
über den soziologischen Rahmen hinaus in Richtung eines urbanisierten und verräumlichten Rahmens.[9]

Alpha City ist eine soziologische Studie. Vielmehr noch aber ist es ein Portrait des Reichtums und des Reichseins in London. Dieser portraithafte Charakter von Atkinsons Abhandlung - immer wieder untermauert von (manchmal etwas klischeehaften) Bildern als auch von (beunruhigenden) Zahlen und Anekdoten - ist sowohl die größte Stärke als auch die größte Schwäche des Buches.

Die vielen Einblicke in das Leben der Superreichen sind interessant; leider bemüht sich Atkinson aber nur wenig um eine systematische Analyse dieser Verhältnisse.[10] Wie Forrest etal. (2018: 91) bemerken, kann eine Analyse, „die den Blick allein auf die superreichen Akteur_innen selbst richtet, das Risiko einer eindimensionalen und zu engen Analyse bergen“. Trotz der Ausweitung der soziologischen Beobachtungen auf jene Akteur_innen, die das Leben der Superreichen in der Stadt ermöglichen, bleiben mehrere sich unweigerlich aufdrängende Fragen unbeantwortet. So etwa die Frage, auf welchen politischen Entscheidungen - besonders seit 2008, aber auch darüber hinausgehend Londons Aufstieg zur Alpha-Stadt fußt? Atkinson sieht die Ursache in einer sich nicht bemerkbar machenden Politikgestaltung (tacit policy making), wie sievon Bürgermeister Boris Johnson auf den Weg gebracht wurde. Eine präzisere Antwort bleibt jedoch aus. Darüber hinaus bedürfte es mehr empirischer Forschung, welche den konkreten Einfluss der Superreichen auf das urbane Gefüge Londons ergründet.

Atkinson spricht an, wie im viktorianischen London die Legitimation der Inanspruchnahme der Stadt durch die Mächtigen und Wohlhabenden um die Idee der Staatsbürgerschaft und der sozialen Verantwortung herum formuliert wurde (vgl. Cherry 1979). Man war sich (wenigstens) des drohenden universellen Leids und der sozialen Unruhen bewusst, die entstünden, würden Investitionen in öffentliche Dienstleistungen und Infrastruktur nicht aufrechterhalten - auch wenn diese Bedenken oft übergangen wurden. Warum, wann und wo ist diese Legitimationsgrundlage verlorengegangen?

Aus der Perspektive der Superreichen, so Atkinson, macht die Privatisierungs- und Individualisierungslogik des neoliberalen Kapitalismus eine quasi-egalitäre Stadtentwicklung überflüssig. Machen sich die Superreichen Sorgen um die Qualität der Krankenhäuser des NHS, können sie auf private Gesundheitsversorgung zurückgreifen. Haben sie Angst vor Kriminalität, so leben die Wohlhabenden in einer Gated Community mit einem umfangreichen, aber von der Umwelt entfremdenden Sicherheitsapparat. Mangelt es den staatlich (unter-)finanzierten Schulen an Qualität, gibt es in London genügend Privatschulen.

Der Erfolg Londons, so Atkinson, wird heute am ,Reichtum' der Stadt gemessen, und wenn Geld das Maß für Reichtum ist, dann ist London tatsächlich die ,beste' Stadt der Welt. Das Narrativ vom monetären Erfolg prägt die Kultur der ,Macher und ,shaker ' der Stadt, die ihre Loyalität ausschließlich den Geldströmen und nicht irgendeiner Idee von Mäzenatentum oder Altruismus verdanken - Werten also, die im vermutlich ebenso inegalitären viktorianischen London wenigstens noch als vorgeschobene Legitimitätsgrundlage für die Inanspruchnahme der Stadt fungierten. Was aber sind die entscheidenden Unterschiede zwischen dem viktorianischen London und dem London von 
heute? Welche Rolle spielt die koloniale Vergangenheit der Stadt London für ihre Gegenwart? Ist London letztlich nur ein Biotop, ein Experimentierlabor, für ein mittlerweile vermutlich schon post-neoliberales Wirtschaftssystem? Ist das London der letzten Dekade - der Dekade als Alpha-Stadt - lediglich ein Vorgeschmack auf die Veränderungen, die in Kürze auch in Beta-Städten wie Paris, Amsterdam, Berlin, Chicago oder Osaka zu beobachten sind? Leider bleiben diese spannenden Fragen unbeantwortet.

Auch normative Fragen drängen sich auf, denn Atkinsons Buch steht an vorderster Front der fortwährenden normativen Debatte über die Zukunft der Stadt. Wie sollen sich urbane Gefüge verändern? Wem soll die Stadt gehören? Wie soll die Stadt ökologisch und ökonomisch funktionieren?[11] Leider finden sich auch hierauf nur wenige Antworten. Vermutlich müssen die Leser_innen ihre Antworten der deskriptiven Kritik an den herrschenden Verhältnissen entnehmen. Es wäre jedoch spannend zu erfahren, welche Stellung London, als die Alpha-Stadt der Gegenwart, in diesen normativen Bewegungen einnimmt.

Etwas naiv wirkt Atkinsons Exposition der bereits 1983 gegründeten Aktivistengruppe Class War, die vorschlug, die unverkauften, leerstehenden Wohnungen im The Shard, dem damals höchsten Wolkenkratzer Europas, zu besetzen[12].

The Shard stand leer, während die Überlebenden des Unglücks im Grenfell Tower[13] nach der Tragödie noch jahrelang wohnungslos blieben, ganz zu schweigen von den rund 4.200 Obdachlosen in der Stadt (Stand: Februar 2020, nach Angaben von Homless.org[14]. Andere Quellen sprechen aktuell von 9.000 Obdachlosen). Die Ungerechtigkeiten in London sind enorm; dennoch ist fraglich, ob das Besetzen eines Hochhauses (wäre es überhaupt möglich) mehr sein kann als ein kläglicher Aufschrei. Es bleibt zu bemängeln, dass Atkinson das Räumliche nur wenig zu politisieren vermag und Alpha City zumeist auf einer deskriptiven Ebene verharrt.[15] Was also tun?

\section{Endnoten}

[1] Forrest etal.(2017) sprechen von „lead cities“oder „global cities“. Diese Begriffsverwendung wird oft als moralische Ächtung verstanden (vgl. Forrest et al. 2018). Mit Bezug auf London ist der Essay „Welcome to „Pikettyville“? Mapping London's alpha territories“ von Burrows et al. (2017) zu nennen.

[2] Für eine Einordung des Begriffs siehe u. a. Rothkopf (2008). Leider ist die Definition von „Superreichtum“ meist vage und immer kontextuell und relational. Eine allgemeine Begriffsdefinition ist nicht zu finden, vielmehr scheint mir der Begriff eine moralisierende Wirkung zu haben. Also solcher wird der Begriff benutzt, um im pejorativen Sinn von Reichtum zu sprechen.

[3] Siehe Webber/Burrows (2016) zu Konflikten in Londoner ,Elitevierteln“

[4] Eine interessante komparative Studie zur Rolle der Mittelschicht in den urbanen Räumen London und Paris, ist z. B. Bacqué et al. (2015). Siehe auch Butler/Robson (2003).

[5] Interessant wäre hier ein Vergleich von Offshore-Unternehmen, welche Land und Immobilien in London kaufen und damit spekulieren, mit britischen Privatpersonen der Mittel- und Oberschicht, die Land und Immobilien an den europäischen Mittelmeerküsten kaufen, um mit diesen zu spekulieren. So fragen sich Forrest et al. (2018: 97): „Ist es lediglich eine Frage der Größenordnung und Intensität? Geht es um Geographien und Zeitlichkeiten? Oder geht es um viel grundsätzlichere Fragen von Macht und sozialer Positionierung? Wie weit sollten wir unseren analytischen Blick ausdehnen?“ 
[6] Für eine geographische Studie über die Superreichen Großbritanniens, siehe Henning/ Dorling (2012).

[7] Von einem,golden passport' wird gesprochen, wenn Staatsbürgerschaft käuflich erworben werden kann. Lea Ypi (2018) zeigt, wie die Idee von der Staatsbürgerschaft als marktfähigem und klassenabhängigem Gut mit dem Kapitalismus verbunden ist und es schon immer war.

[8] Tatsächlich kommt es durch Enteignungen (und eine Politik, die diese bewilligt), wie Sassen (2014) beschreibt, zu einer neuen Form der primitiven Akkumulation im Marxschen Sinn.

[9] Leider mangelt es Atkinsons Buch an einer präzisen Definition der ,Stadtelite‘. Siehe Savage (2015) für einen der wenigen Versuche, Elitensoziologie und Urbanistik zusammenzuführen.

[10] Um eine differenziertere Einordung sorgen sich Beaverstock/Faulconbridge (2013).

[11] Mit dem Bezug auf London sind hier Richard Floridas 2009 erschienes Werk Who's your City? oder Merryfield (2014) zu nennen, welche eben die oben genannten Fragen aufwerfen.

[12] https://www.london-se1.co.uk/news/view/9540 (letzter Zugriff am 19.10.2020).

[13] Am 14 Juni 2017 brach in der Nacht ein Feuer im Grenfell Tower, einem Sozialwohnungsobjekt im Londoner Stadtteil Kensington, aus, bei dem 72 Menschen starben und genauso viele schwer verletzt wurden. Der Brand breitete sich schnell über die Wärmedämmung der Fassade aus - die Stadt London missachtete die zahlreichen Hinweise auf die Gefahrenlage. Der Fall wurde im Anschluss von Scotland Yard untersucht. Ein Gerichtsurteil wurde noch nicht verlesen. Der Grenfell Tower ist zum traurigen Symbolbild der Londoner Wohnungspolitik geworden: Sozialhilfeempfänger verbrennen, die High Society Kensigtons sieht dabei zu.

[14] https://www.homeless.org.uk/facts/homelessness-in-numbers/rough-sleeping/roughsleeping-our-analysis (letzter Zugriff am 19.10.2020).

[15] Als Messlatte des normativen Anspruchs kann zum Beispiel die Arbeit von Daniel Mullis (2014) gesehen werden, welche an das Konzept der Radikalen Demokratietheorie nach Chantal Mouffe und Ernesto Laclau anknüpft. Vgl. etwa Carstensen-Egwuom (2014) sowie Beebeejaun (2017).

\section{Autor innen}

Adrian Kreutz forscht in der Politischen Theorie. Aktuell arbeitet er an seiner Promotion zum Politischen Realismus.

adrian.kreutz@politics.ox.ac.uk

\section{Literatur}

Action on Empty Homes (2020): In: Pretty vacant report (28. April 2020). https://www. actiononemptyhomes.org/Handlers/Download.ashx?IDMF=24ace1b7-b428-4fee-8dcc6a7638f32eaa (letzter Zugriff am 20.10.2020).

Atkinson, Rowland (2006): Padding the bunker: Strategies of middle-class disaffiliation and colonisation in the city. In: Urban Studies 43/4, 819-832.

Atkinson, Rowland (2020): Alpha City. How London was captured by the super-rich. London: Verso.

Bacqué, Marie-Helene / Bridge, Gray / Butler, Tim / Charmes, Eric / Fijalkow, Yankel / Jackson, Emma / Launay, Lydie / Vermeersch, Stéphanie (2015): The middle classes and the city: A study of Paris and London. London: Palgrave.

Bauman, Zygmunt (2013): Does the richness of the few benefit us all? Cambridge/Malden, MA: Polity Press. 
Beaverstock, Jonathan V. (2012): The privileged world city: Private banking, wealth management and the bespoke servicing of the global super rich. In: Ben Derudder / Michael Hoyler / Peter Taylor / Frank Witlox (Hg.), International handbook of globalization and world cities. Cheltenham/Northampton: Edward Elgar, 378-389.

Beaverstock, Jonathan V./ Faulconbridge, James R. (2013): Wealth segmentation and the mobilities of the super-rich: A conceptual framework. In: Thomas Birtchnell / Javier Caletrio-Garcera (Hg.), Elite Mobilities. London: Routledge, 40-61.

Beaverstock, Jonthan V. / Smith, Richard G. / Taylor, Peter (1999): A roster of world cities. In: Cities 16/6,445-458.

Beebeejaun, Yasminah (2017): Gender, urban space, and the right to everyday life. In: Journal of Urban Affairs 39/3, 323-334.

Burrows, Roger / Webber, Richard / Atkinson, Rowland (2017): Welcome to ,Pikettyville'? Mapping London's alpha territories. In: The Sociology Review 65/2: 184-201.

Butler, Tim / Robson, Gerry (2003): London calling: The middle classes and the remaking of Inner London. London: Bloomsbury.

Carstensen-Egwuom, Inken (2014): Connecting intersectionality and reflexivity. Methodological approaches to social positionalities. In: Erdkunde 68/4, 265-276.

Cherry, Gordon (1979): The town planning movement and the late victorian city. In: Transactions of the Institute of British Geographers 4/2, 306-319.

Florida, Richard (2009): Who's your city? London: Basic Books/Random House.

Forrest, Ray / Koh, Sin Yeh / Wissink, Bart (Hg.) (2017): Cities and the super-rich. Real estate, elite practices and urban political economies. New York: Palgrave Macmillan.

Forrest, Ray / Koh, Sin Yeh / Wissink, Bart (Hg.) (2018): Hypergespaltene Städte und die ,unmoralischen' Superreichen - Fünf abschließende Fragen. In: sub \urban. zeitschrift für kritische stadtforschung 6/2-3, 91-104.

Goldfarb, Michael (2013): London's great exodus. In: New York Times, 12.10.2013. http://www.nytimes.com/2013/10/13/opinion/sunday/londons-great-exodus. html?pagewanted=all\&_r=o (letzter Zugriff am 30.8.2020).

Henning, Benjamin / Dorling, Danny (2012): Mapping Britain's super-rich. In: Political Insight $3 / 3,42$.

Koh, Sin Yeh / Wissink, Bart / Forrest, Ray (2016): Reconsidering the super-rich: Variations, structural conditions, and urban consequences. In: Ian Hay, Jonathan V. Beaverstock (Hg.), International handbook on wealth and the super-rich. Cheltenham: Edward Elgar Publishing, 18-40.

Merrifield, Andy (2014): The new urban question. London: Pluto Press.

Mikes, George (1951): Down with everybody. London: Allan Wingate.

Mullis, Daniel (2014): Recht auf die Stadt - Von Selbstverwaltung und radikaler Demokratie. Münster: Unrast.

Rothkopf, David (2008): Superclass. New York: Little, Brown and Company.

Sassen, Saskia (2014): Expulsions: brutality and complexity in the global economy. Cambridge: Harvard University Press.

Savage, Mike (2015): Social class in the 21st century. London: Pelican Books.

Sayer, Andrew (2015): Why we can't afford the rich. Bristol/Chicago: Policy Press.

Webber, Richard / Burrows, Roger (2016): Life in an alpha territory: Discontinuity and conflict in an elite London ,village‘. In: Urban Studies 53/15, 3319-3154.

Ypi, Lea (2018): Borders of class: Migration and citizenship in the capitalist state. In: Ethics \& Public Affairs 32/2, 141-152. 